\title{
Annuaire suisse de politique de
}

\section{L'impact du tiers monde sur les postes de travail en Suisse}

Guido Pult

\section{(2) OpenEdition}

Édition électronique

URL : http://journals.openedition.org/aspd/1022

DOI : $10.4000 /$ aspd. 1022

ISSN : 1663-9669

Éditeur

Institut de hautes études internationales et du développement

\section{Édition imprimée}

Date de publication : 1 janvier 1983

Pagination : 227-245

ISSN : 1660-5934

\section{Référence électronique}

Guido Pult, «L'impact du tiers monde sur les postes de travail en Suisse », Annuaire suisse de politique de développement [En ligne], 3 | 1983, mis en ligne le 29 novembre 2012, consulté le 08 septembre 2020. URL : http://journals.openedition.org/aspd/1022 ; DOI : https://doi.org/10.4000/aspd.1022 


\title{
L'IMPACT DU TIERS MONDE SUR LES POSTES DE TRAVAIL EN SUISSE
}

\author{
Guido Pult
}

Zusammenfassung: Auswirkungen der Entwicklung in der Dritten Welt auf die Arbeitsplätze in der Schweiz

Dieser Artikel zeigt auf, dass der Saldo der in der Schweiz an die Dritte Welt gekoppelten Arbeitsplätze zwischen 1973 und 1980 nicht variiert hat. So sind die von der Uhren-, Bekleidungs- und Textilindustrie erlittenen Verluste grösstenteils durch die in den anderen Sektoren erwirtschafteten Gewinne ausgeglichen worden. Allerdings lassen sich gewisse Ungleichheiten feststellen, wenn man einzelne Regionen und Berufssparten untersucht. Das gilt insbesondere für die von der Uhrenindustrie beherrschten Regionen. Die Gesamtzahl der Arbeitsplätze in der Schweiz ist zwar gesunken; dies geht aber also nur bedingt auf Entwicklungen in der Dritten Welt zurück.

\section{INTRODUCTION}

De 1973 à 1976 l'économie suisse a perdu un nombre impressionnant de postes de travail: 285.000 , soit le $9 \%$ (1). Dans les années suivantes et jusqu'à 1981, environ la moitié de ces postes a été récupérée. Mais même en cette période, le niveau de l'emploi de certaines branches n'a cessé de fléchir. Puisquil s'agit de branches - l'horlogerie, les textiles, I'habillement - qui subissent la concurrence des pays à bas salaires, la question est souvent posée de savoir dans quelle mesure la concurrence du Tiers Monde est responsable de la dégradation de l'emploi en Suisse. C'est la question qui nous intéresse ici. Les limites que nous nous sommes fixées excluent tout recours à des méthodes $d^{\prime}$ analyse qui ne soient pas élémentaires. II ne s'agit que d'indiquer quelques points de repère que les statistiques et la littérature suggèrent, le long d'un chemin difficile à tracer.

La documentation existante ne permet d'appréhender qu'une partie des relations économiques Suisse - Tiers Monde. Ainsi, dans ce qui suit, il ne sera question pour l'essentiel que des relations commerciales 
entre ces deux partenaires et des exportations de ceux-ci sur le marché mondial. En particulier, rien ne sera dit sur les exportations suisses vers les marchés tiers, de biens qui sont utilisés dans la production de marchandises destinées au Tiers Monde; de même seront exclus les biens et les services produits dans le Tiers Monde mais incorporés à des marchandises vendues à la Suisse par d'autres pays.

La période à laquelle nous ferons référence va de 1973 à 1980. Pour les années antérieures, le chômage n'est pas aigu; quant aux années plus récentes, caractérisées par l'apparition d'une nouvelle récession, les sources de documentation sont nettement moins abondantes.

\section{LE COMMERCE EXTERIEUR SUISSE - TIERS MONDE}

Le tableau 1 nous donne un aperçu du commerce extérieur de la Suisse avec le Tiers Monde pour la période qui nous intéresse.

Tableau 1

\section{COMMERCE SUISSE-TIERS MONDE* \\ (Mio de Fr.)}

\begin{tabular}{|c|c|c|c|c|c|}
\hline Année & Exportations & Importations & $(1)-(2)$ & (1) $\times 100$ & (1) $\times 1$ \\
\hline & (1) & (2) & & $(1)+(2)$ & PNB \\
\hline 1973 & 4370,4 & 2616,7 & 1753,7 & 62,5 & 3,2 \\
\hline 1974 & 5549,2 & 3821,9 & 1727,3 & 59,2 & 3,8 \\
\hline 1975 & 5972,8 & 3095,5 & 2877,3 & 65,9 & 4,1 \\
\hline 1976 & 6696,8 & 3320,5 & 3376,3 & 66,9 & 4,6 \\
\hline 1977 & 8079,1 & 4108,6 & 3970,5 & 66,3 & 5,3 \\
\hline 1978 & 8056,4 & 3266,1 & 4790,3 & 71,2 & 5,1 \\
\hline 1979 & 7850,4 & 3718,6 & 4131,8 & 67,8 & 4,8 \\
\hline 1980 & 9064,5 & 5374,1 & 3690,4 & 62.7 & 5,1 \\
\hline
\end{tabular}

Source des données de base: Statistique annuelle du commerce extérieur de la Suisse; Annuaire statistique de la Suisse.

* Tiers Monde: L'ensemble des pays "non planifiés" qui n'appartiennent pas à l'OCDE et sans I'Afrique du Sud et Israël. 
II est à remarquer que le concept de Tiers Monde que nous employons ici diffère de celui des statistiques officielles suisses. En effet, contrairement à ce dernier, il ne comprend pas la Yougoslavie, Israël et le Vietnam, qu'il nous semble judicieux d'intégrer plutôt aux régions développées ou aux économies socialistes, conformément aux publications des Nations Unies. La différence n'est pas négligeable, car de la sorte le solde annuel de la balance s'amenuise dans une mesure qui souvent est de l'ordre de $20-30 \%$, du fait de l'excédent très important réalisé dans les échanges avec la Yougoslavie et Israël. Néanmoins, comme le montre le tableau 1 , le solde actif reste considérable.

II arrive assez souvent que l'existence d'un tel excédent suscite des malentendus. En passant, et pour éliminer ce danger, remarquons $\mathrm{d}^{\prime}$ abord que ce montant est fortement influencé par le fait que la Suisse, contrairement à beaucoup de pays de l'OCDE, n'importe une grande partie de son pétrole qu'après raffinage dans un pays industrialisé. II s'ensuit que dans les statistiques ces importations apparaissent comme des livraisons de ces derniers pays. En outre et surtout: le déséquilibre commercial ne signifie nullement qu'un pays s'enrichit aux dépens de l'autre, de même que par exemple le boucher dont les ventes au boulanger sont plus élevées que ses achats de pains, n'exploite pas nécessairement ce dernier. L'escroquerie ne subsiste que si le rapport du prix des biens vendus aux prix des biens achetés n'est pas «juste».

Le malentendu peut être favorisé par l'habitude d'adopter des schémas keynésiens (2). D'après le plus courant de ces schémas, en effet, un accroissement de l'excédent commercial de la nation comporte une augmentation du revenu national, et de façon symétrique, une réduction du premier provoque celle de l'autre. Mais ce modèle repose sur I'hypothèse que l'équipement productif est sous-utilisé; en outre il n'envisage pas l'augmentation du revenu qui fait suite à l'augmentation de la capacité de production. Or, dans le Tiers Monde, souvent l'équipement au lieu d'être abondant fait défaut et le problème de l'accroissement de la capacité de production est crucial. Dans ces conditions, d'une part le déficit de la balance commerciale n'a pas d'effets négatifs sur la demande interne et donc sur le revenu national courant; d'autre part il importe de considérer que, dans la mesure où le déficit se traduit par I'achat de biens d'équipement, il peut permettre l'accroissement du revenu national futur. 


\section{IMPORTATIONS DE SUBSTITUTION IMPORTATIONS COMPLEMENTAIRES}

Du point de vue de l'impact sur les postes de travail, il y a une asymétrie importante entre les exportations et les importations: tandis qu'en principe toute exportation exerce un effet positif sur l'emploi, on ne peut pas affirmer que toute importation affecte ce dernier négativement. II faut en effet distinguer entre importations de substitution et complémentaires. Les premières portent sur les biens dont I'achat se fait au détriment de produits nationaux similaires. Les deuxièmes, par contre, concernent les biens qui ne sont pas produits dans le pays: matières premières, mais aussi biens d'équipement et de consommation. II est clair que ce genre d'importations est une condition nécessaire pour le maintien et l'accroissement d'un certain nombre d'emplois.

Pour la Suisse, les statistiques existantes permettent aisément de structurer les importations depuis le Tiers Monde selon les catégories du tableau 2.

Tableau 2

IMPORTATIONS DE LA SUISSE DEPUIS LE TIERS MONDE SELON LE GENRE DE MARCHANDISES

(Mio de Fr.)

\begin{tabular}{lcrcccc} 
Année & $\begin{array}{l}\text { Mat. premières } \\
\text { et demi-produits }\end{array}$ & Energie & $\begin{array}{l}\text { Biens d' } \\
\text { équipement }\end{array}$ & $\begin{array}{l}\text { Biens de } \\
\text { consomm. }\end{array}$ & Total & $\begin{array}{l}\text { Total mnins } \\
\text { énergie }\end{array}$ \\
\hline 1973 & 1183,9 & 663,3 & 22,2 & 747,3 & 2616,7 & 1953,4 \\
1974 & 1318,2 & 1635,5 & 23,4 & 844,8 & 3821,9 & 2186,4 \\
1975 & 1224,2 & 1091,5 & 33,3 & 746,5 & 3095,5 & 2004,0 \\
1976 & 1450,4 & 990,5 & 36,3 & 843,3 & 3320,5 & 2330,0 \\
1977 & 1841,0 & 1063,0 & 28,4 & 1176,2 & 4108,6 & 3045,6 \\
1978 & 1388,7 & 740,6 & 34,2 & 1102,6 & 3266,1 & 2525,5 \\
1979 & 1480,8 & 1125,9 & 35,2 & 1076,7 & 3718,6 & 2592,7 \\
1980 & 2282,7 & 1618,6 & 43,7 & 1429,1 & 5374,1 & 3755,5
\end{tabular}

Source des données de bases: Statistique annuelle du commerce extérieur de la Suisse. 
Clairement dans le cas de la Suisse les produits énergétiques et les matières premières importées doivent être considérés comme des biens complémentaires. Et puisque les importations de biens d'équipement sont négligeables, on peut affirmer que la concurrence du Tiers Monde est limitée à une partie des biens semi-produits et des biens de consommation. La valeur des importations de substitution est donc inférieure au chiffre de la dernière colonne du tableau, laquelle nous donne le montant des importations après avoir éliminé les produits énergétiques.

\section{POSTES DE TRAVAIL LIES AU COMMERCE EXTERIEUR AVEC LE TIERS MONDE : BILAN}

L'impact du commerce extérieur sur l'emploi concerne les entreprises qui produisent les biens exportés ou exposés à la concurrence étrangère, mais non seulement elles. II affecte aussi les entreprises produisant les biens achetés par celles-ci et par leurs bénéficiaires de revenu, et ainsi de suite. Afin d'évaluer l'ensemble des postes de travail liés au commerce extérieur, il faudrait donc connaître la structure, selon le genre de marchandise, des achats, d'une part de toutes les branches touchées et de l'autre, des différents groupes de bénéficiaires de revenus. Le premier type de renseignements est livré par les tableaux des échanges interdustriels, qui pour la Suisse ne sont pas encore disponibles. Dans ces conditions, bien qu'on soit mieux lotis pour l'autre catégorie de renseignements, les calculs ne peuvent être que très approximatifs.

Pour estimer le nombre de postes de travail directs liés aux exportations on peut simplement diviser le montant des exportations des différentes branches économiques par le chiffre d'affaires par travailleur de ces dernières. C'est la méthode utilisée par Gerster (1976) dans une riche contribution de pionnier. Cet auteur estime qu'en 1974, les emplois directs liés aux exportations suisses vers le Tiers Monde s'élevaient à environ 100.000. Toutefois le concept de Tiers Monde qu'il retient est celui des statistiques officielles que nous avons évoqué avant. En outre l'estimation du chiffre d'affaires par travailleur apparaît trop faible au regard des données - à l'époque non encore disponibles - relevées par le recensement des entreprises de 1975. En utilisant ces dernières données (3) et la même structure des exportations que Gerster, mais par rapport au concept de Tiers Monde que nous avons adopté ici, on peut affirmer que les postes de travail en question étaient en 1975 d'environ la moitié, c'est-à-dire 50.000.

L'évaluation de l'impact des importations est plus délicate. A la difficulté, déjà signalée, d'isoler les importations de substitution, s'ajoute le fait que l'adoption de la méthode que nous venons d'appliquer aux 
exportations (division par le chiffre d'affaires par tête) comporte une marge d'erreur nettement plus ample. Cette méthode revient à supposer que, par exemple, l'importation d'un million de francs de chaussures diminue $d$ 'autant les ventes de chaussures suisses; mais si celles-là sont nettement meilleur marché, et de qualité similaire, la réduction des ventes risque de dépasser le montant en question. Cependant, en pratique, les renseignements manquent pour recourir à une méthode moins grossière.

Gerster (1976) en utilisant la méthode en question, estime que pour 1974 le nombre de postes de travail potentiels supprimés par la concurrence du Tiers Monde s'élevait à environ 50.000. A l'égard de ce chiffre on peut formuler les mêmes remarques que nous avons vues à propos de l'impact des exportations. En outre dans ce calcul on considère toutes les importations, sans déduire celles à caractère complémentaire. En éliminant les produits énergétiques et en procédant comme nous l'avons fait pour les exportations, on trouve que pour 1975 I'ordre de grandeur du nombre recherché est de 20.000. Soulignons qu'à cause de l'impossibilité d'isoler les importations de substitution, il s'agit d'un plafond. Nous arrivons ainsi à la conclusion qu'en tenant compte des emplois supprimés par la concurrence, les emplois directs liés au commerce avec le Tiers Monde, en 1975, se situaient entre 30.000 (cas où le plafond que nous venons d'évoquer est atteint) et 50.000 (cas où il n'y aurait que des importations complémentaires).

Pour se faire une idée sur la création d'emplois indirects, on peut commencer par constater qu'en 1975 environ la moitié du chiffre d'affaires des entreprises était consacré à l'achat de biens intermédiaires et que la propension à consommer des biens et services domestiques était proches de 0,4 . Dans ces conditions et de façon très prudente, on peut affirmer que les effets indirects sont du même ordre de grandeur que les effets directs. L'ordre de grandeur des postes de travail directs et indirects liés au commerce avec le Tiers Monde peut ainsi être estimé à l'intérieur d'une fourchette de 60.000 à 100.000 .

\section{POSTES DE TRAVAIL LIES AU COMMERCE AVEC LE TIERS MONDE : VARIATIONS DU BILAN}

Jusqu'ici il a été question d'un bilan des postes de travail par rapport à une année particulière. Dans l'optique de l'étude des causes du chômage et des fluctuations de l'emploi, ce n'est pas un tel bilan qui nous intéresse, mais sa variation. Sur la base des tableaux 1 et 2 on constate que dans la période 1975-1980, l'excédent des exportations sur le plafond des importations de substitution a augmenté de $34 \%$. Ce taux est à comparer à la variation du chiffre d'affaires par tête, qui en 
moyenne peut être évalué à $20 \%$ (somme de l'accroissement des prix, d'après le déflateur du PIB, et de la productivité). On peut ainsi estimer que pendant la période 1975-1980 la variation des éléments de la fourchette des postes de travail liés au Tiers Monde, a été légèrement positive. Ceci n'exclut pas qu'à l'intérieur de la fourchette, le nombre effectif - que nous ne connaissons pas - des postes de travail ait diminué.

En tout cas, pendant la période 1973-1980, dans l'ensemble des postes de travail disponibles en Suisse, la fraction liée au Tiers Monde a augmenté sensiblement. On peut le déduire du tableau 1, où l'on remarque que le rapport des exportations sur le produit national brut, qui était de $3,2 \%$ en 1973, après 1977 s'est toujours situé autour de $5 \%$. Pendant la même période, comme on le voit dans le tableau 3 , la part dans les exportations totales des exportations vers le Tiers Monde a progressé de 15,4 à $18,8 \%$. Le même tableau nous montre que cet accroissement est entièrement dû au commerce avec les pays exportateurs de pétrole (PEP): à l'égard des autres pays du Tiers Monde, la part des exportations a été d'une remarquable stabilité.

Tableau 3

STRUCTURE DU COMMERCE EXTERIEUR SUISSE SELON LES REGIONS $\begin{array}{lllllllll}\text { Exportations } & 1973 & 1974 & 1975 & 1976 & 1977 & 1978 & 1979 & 1980\end{array}$

Economies de marché

développées 79,6 77,7 75,1 75,2 74,3 75,2 77,3 77,1

Economies planifiées 5,0

$$
5,6
$$

Tiers Monde *

15,4

16,7

18,6

18,9

20,2

19,8

18,2

18,8

Pays export.

de pétrole

$\begin{array}{llllllll}3,6 & 4,4 & 6,7 & 7,8 & 8,4 & 8,2 & 6,4 & 7,0\end{array}$

Autres

\begin{tabular}{llllllll}
11,8 & 12,3 & 11,9 & 11,1 & 11,8 & 11,6 & 11,8 & 11,8 \\
\hline 100,0 & 100,0 & 100,0 & 100,0 & 100,0 & 100,0 & 100,0 & 100,0
\end{tabular}




\section{Importations}

Economies de marché

développées

$\begin{array}{llllllll}90,2 & 88,1 & 87,9 & 87,0 & 86,6 & 88,3 & 88,1 & 86,9\end{array}$

Economies

planifiées

$\begin{array}{llllllll}2,4 & 2,8 & 2,8 & 3,6 & 3,6 & 3,8 & 4,1 & 4,3\end{array}$

Tiers Monde* dont:

$\begin{array}{llllllll}7,4 & 9,1 & 9,2 & 9,3 & 9,8 & 7,9 & 7,7 & 8,9\end{array}$

Pays export.

de pétrole

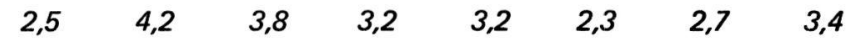

Autres

\begin{tabular}{rrrrrrrr}
4,9 & 4,9 & 5,4 & 6,1 & 6,6 & 5,6 & 5,0 & 5,5 \\
\hline 100,0 & 100,0 & 100,0 & 100,0 & 100,0 & 100,0 & 100,0 & 100,0
\end{tabular}

Source des données de base: Nations Unies, Annuaire statistique du commerce international;calculs effectués sur la base de valeurs en dollars.

${ }^{*} Y$ compris la Turquie 


\section{COMMERCE AVEC LES "NOUVEAUX PAYS INDUSTRIALISES" (NPI)}

On peut se demander dans quelle mesure la concurrence du Tiers Monde n'a pas été le fait de ceux qu'on appelle souvent les "Nouveaux pays industrialisés" (NPI) (4). Entre 1973 et 1980 les importations suisses depuis ces pays ont presque doublé (cf. le tableau 4) tandis que les importations depuis les autres pays du Tiers Monde nonappartenant aux PEP ne progressaient que d'environ $60 \%$. Toutefois simultanément les exportations vers les NPI augmentaient de $70 \%$, ce qui a permis à la Suisse de conserver un solde actif considérable. En effet, ce solde a augmenté de $30 \%$, une mesure proche de la somme de la variation des indices des prix et de la productivité. Ce qui suggère qu'en gros les postes de travail supprimés à cause des importations ont été compensés par ceux qui ont été gagnés par l'augmentation des exportations.

Tableau 4

COMMERCE EXTERIEUR DE LA SUISSE AVEC LES “NOUVEAUX PAYS INDUSTRIALISES"

(Mio de Fr.)

Année

Exportations

Importations

(1) - (2)

(1) $\times 100$

(1)

(2)

$(1)+(2)$

\begin{tabular}{lrrrr}
\hline 1973 & 1703,0 & 715,3 & 987,7 & 70,4 \\
1974 & 2038,9 & 788,4 & 1250,5 & 72,1 \\
1975 & 1787,7 & 769,1 & 1018,6 & 69,9 \\
1976 & 2004,1 & 924,5 & 1079,6 & 68,4 \\
1977 & 1970,1 & 1129,2 & 840,9 & 63,6 \\
1978 & 2150,8 & 922,9 & 1227,9 & 70,0 \\
1979 & 2533,3 & 1011,3 & 1522,0 & 71,5 \\
1980 & 2696,9 & 1414,7 & 1282,2 & 65,6
\end{tabular}

Source des données de base: Statistique annuelle du commerce extérieur de la Suisse.

* Argentine, Brésil, Hongkong, Mexique, Singapour, Corée du Sud, Taiiwan 


\section{PENETRATION SELON LES BRANCHES}

Un effet d'identification des branches qui sont particulièrement exposées à la concurrence du Tiers Monde a été effectué notamment par Hollenstein (1979 et 1980). Les calculs de cet auteur permettent d'établir le tableau 5 .

Tableau 5

\section{PART DES IMPORTATIONS DES PAYS A BAS SALAIRES* DANS LA DEMANDE INTERNE** SUISSE.}

(En pourcentage)

Branches

Alimentation, boissons, tabac

Textiles

Habillement

Bois

Papier

Imprimerie et édition

Cuir, caoutchouc, mat. plastiques

Chimie

Produits minéraux non métalliques, sauf pétrole et charbon

Métaux

Véhicules

Machines et matériel

Horlogerie

Industrie manufacturière

\begin{tabular}{llll}
$1960 / 62$ & $1964 / 66$ & 1970 & 1975 \\
\hline 2,0 & 2,2 & 2,9 & 2,2 \\
2,5 & 3,3 & 3,7 & 5,6 \\
0,5 & 1,1 & 2,8 & 6,4 \\
1,3 & 1,6 & 1,8 & 1,6 \\
0,05 & 0,1 & 0,4 & 0,7 \\
0,0 & 0,05 & 0,05 & 0,1 \\
0,4 & 0,6 & 0,9 & 1,6 \\
1,2 & 1,7 & 1,5 & 1,8 \\
0,1 & 0,15 & 0,25 & 1,5 \\
1,1 & 0,9 & 1,0 & 0,6 \\
0,0 & 0,05 & 0,05 & 0,5 \\
0,05 & 0,1 & 0,2 & 0,8 \\
0,05 & 0,1 & 0,6 & 1,4 \\
& & &
\end{tabular}

1,1

1,3

1,5

2,0

Source: Hollenstein, 1979

* Ce concept est plus large que celui de Tiers Monde. Par rapport à la définition de ce dernier que nous avons adopté, il comprend aussi Portugal, Espagne, Grèce, Malte, Gibraltar, Albanie, Bulgarie, Roumanie, Yougoslavie, Afrique du Sud, Chine.

** Production domestique moins exportations plus importations. 
On constate que dans la période 1960-1975 et pour l'ensemble des branches industrielles, le taux de pénétration des pays à bas salaires (dont la composante Tiers Monde est essentielle) a presque doublé. L'augmentation n'a été plus faible que dans I'alimentation, la chimie, les métaux et le bois.

Ces chiffres témoignent des transformations structurelles qui sont en cours dans les pays à bas salaires. Mais ils ne doivent pas faire oublier qu'en 1975 le taux de pénétration de ces pays restait très faible: $2 \%$ en moyenne, seuil que seuls les textiles et l'habillement dépassaient nettement.

\section{CONCURRENCE SUR LE MARCHE MONDIAL}

Jusqu'à maintenant nous $\mathrm{n}^{\prime}$ avons pris en compte que la concurrence du Tiers Monde sur le marché suisse. Quant à la concurrence que celuici exerce sur le marché mondial, commençons par considérer les données de caractère global que nous fournit le tableau 6 .

On remarque que, de 1973 à 1980, la tranche de la Suisse dans les exportations mondiales s'est réduite d'environ un dixième. Au cours de cette période, ce n'est qu'en 1978 que la tranche a été inférieure à celle du début. Exception qu'on peut attribuer à la forte appréciation du taux de change (le tableau se rapporte à des montants en dollars). Ce rétrécissement correspond de très près à celui concernant l'ensemble des pays développés à économie de marché. En effet, en 1980 le rapport entre les exportations suisses et de ces derniers pays était de 2,4\%, comme en 1973.

Dans la même période, la part dans les exportations mondiales des pays à économie planifiée a diminué en proportion analogue. Ainsi le seul gagnant a été le Tiers Monde. Cependant, l'accroissement de la part de ce dernier, qui a été de presque $50 \%$, est entièrement imputable à la hausse du prix du pétrole (les pays exportateurs de pétrole ont gagné 8,8 points, ce qui correspond aux points gagnés par l'ensemble du Tiers Monde). Et puisque, pour la plupart des pays développés, le pétrole est un bien complémentaire plutôt que de substitution, on peut conclure que, globalement et avec toutes les réserves liées au caractère grossier de l'indicateur en question, la capacité concurrentielle de ces pays, ainsi que celle de la Suisse, est restée intacte. La pression accrue exercée par les NPI, pour ce qui concerne la Suisse, s'est traduite par une réduction du rapport entre ses exportations et celles de ce groupe de pays, qui a passé de 44,6 à $35 \%$. En même temps, à l'égard des autres pays non pétroliers du Tiers Monde, le même indicateur est resté pratiquement stable, malgré l'accroissement très net vers les "pays les moins avancés» (de 330 à $490 \%$ ). 
Tableau 6

STRUCTURE DES EXPORTATIONS MONDIALES SELON LES PAYS

(En pourcentage)

\begin{tabular}{|c|c|c|c|c|c|c|c|c|}
\hline & 1973 & 1974 & 1975 & 1976 & 1977 & 1978 & 1979 & 1980 \\
\hline \multicolumn{9}{|l|}{ Pavs développés à économie } \\
\hline de marché & 70,8 & 64,6 & 66,0 & 64,7 & 64,6 & 67,0 & 65,2 & 63,1 \\
\hline Amérique & 16,8 & 15,6 & 16,0 & 15,4 & 14,4 & 14,5 & 14,3 & 14,2 \\
\hline Europe & 44,8 & 40,2 & 41,5 & 40,4 & 41,0 & 42,9 & 42,5 & 40,4 \\
\hline Japon & 6,4 & 6,6 & 6,4 & 6,8 & 7,1 & 7,5 & 6,3 & 6,5 \\
\hline Tiers Monde* & 19,2 & 26,9 & 24,3 & 25,9 & 25,8 & 23,3 & 25,6 & 28,0 \\
\hline Pays exp. de pétrole & 8,1 & 16,2 & 14,1 & 15,0 & 14,6 & 12,3 & 14,5 & 16,9 \\
\hline Nouv. pays ind. ${ }^{*}$ & 3,7 & 3,3 & 3,2 & 3,7 & 4,0 & 4,1 & 4,1 & 4,2 \\
\hline Pays moins avancés & 0,5 & 0,4 & 0,4 & 0,4 & 0,4 & 0,3 & 0,3 & 0,3 \\
\hline Autres pays & 6,9 & 7,0 & 6,6 & 6,8 & 6,8 & 6,6 & 6,7 & 6,6 \\
\hline Pays à écon. planifiées & 10,0 & 8,5 & 9,7 & 9,4 & 9,6 & 9,7 & 9,2 & 8,9 \\
\hline Monde & 100,0 & 100,0 & 100,0 & 100,0 & 100,0 & 100,0 & 100,0 & 100,0 \\
\hline $\begin{array}{l}\text { Suisse } \\
\text { Exp. suisses } \times 100\end{array}$ & 1,65 & 1,42 & 1,48 & 1,49 & 1,57 & 1,81 & 1,61 & 1,47 \\
\hline $\begin{array}{l}\text { Exp. autres pays à } \\
\text { écon. de marché }\end{array}$ & 2,4 & 2,2 & 2,3 & 2,4 & 2,5 & 2,8 & 2,5 & 2,4 \\
\hline Exp. suisses $\times 100$ & & & & & & & & \\
\hline$\overline{\text { Exp. NPI }}$ & 44,6 & 43,0 & 46,3 & 40,3 & 39,3 & 44,1 & 39,3 & 35,0 \\
\hline Exp. suisses $\times 100$ & 330 & 350 & 370 & 370 & 390 & 600 & 540 & 490 \\
\hline
\end{tabular}

Source des données de base: Nations Unies, Manuel de statistique du commerce international et du développement, 1981

* Cf. note du tableau 3

* Sans le Mexique (incorporé aux pays exportateurs de pétrole) et Taiwan 
PART DES EXPORTATIONS DE LA SUISSE DANS LES EXPORTATIONS DES PAYS A ECONOMIE DE MARCHE: BRANCHES DONT LA PART EN 1980 ETAIT PLUS FAIBLE QU'EN 1973 ET DONT LE MONTANT EN 1976/77 DEPASSAIT LES 100 MILLIONS DE DOLLARS.

\begin{tabular}{|c|c|c|c|c|c|}
\hline Branches & $\begin{array}{c}\text { Mio. de \$ } \\
\quad(1980)\end{array}$ & $\begin{array}{c}1973 \\
\%\end{array}$ & $\begin{array}{c}1976 \\
\%\end{array}$ & $\begin{array}{c}1980 \\
\%\end{array}$ & $\begin{array}{l}\text { Principaux } \\
\text { pays dont } \\
\text { la part à } \\
\text { augmentée }\end{array}$ \\
\hline Fromage et caillebotte & 289 & 8,7 & 8,0 & 7,3 & $\mathrm{D}, \mathrm{DK}$ \\
\hline Tabacs fabriqués & 104 & 10,5 & 6,8 & 2,9 & $\begin{array}{l}\text { USA, GB,NL } \\
\text { D }\end{array}$ \\
\hline Composés fonctions azotées & 550 & 10,4 & 11,1 & 10,1 & USA,GB,B \\
\hline Composés organo-minéraux & 843 & 20,5 & 17,9 & 16,0 & IRL,USA \\
\hline Colorants organiques, laques & 719 & 27,0 & 25,1 & 23,2 & Japon,GB,I \\
\hline Produits médicinaux & 1615 & 12,5 & 12,3 & 11,7 & USA, F,GB \\
\hline Huiles essentielles & 200 & 12,5 & 13,3 & 12,2 & $\begin{array}{l}\text { USA,GB,D, } \\
\text { IRL }\end{array}$ \\
\hline Pesticides, désinfectants & 317 & 9,9 & 9,2 & 7.4 & $\begin{array}{l}\text { USA,B,F, } \\
\text { GB }\end{array}$ \\
\hline Pompes & 344 & 5,3 & 5,4 & 4,0 & Japon,GB,I \\
\hline Machines outils & 1049 & 9,7 & 9,3 & 9,4 & Japon,I,E \\
\hline Appareils coupure & 728 & 6,1 & 6,0 & 5,8 & Japon, GB \\
\hline Instruments de mesure & 897 & 6,2 & 6,0 & 6,0 & USA,GB \\
\hline Horlogerie & 2121 & 47,9 & 34,8 & 28,2 & $\begin{array}{l}\text { Hongkong, } \\
\text { Japon, } \\
\text { Corée }\end{array}$ \\
\hline Imprimés & 284 & 4,4 & 3,4 & 3,6 & $D, I, E$ \\
\hline
\end{tabular}

Source des données de base: Nations Unies, Annuaire statistique du commerce international, vol. II

* Pour les pays européens: symbole des plaques minéralogiques 
L'impression de stabilité de la capacité concurrentielle est confirmée par I'analyse par branches. Dans la statistique des parts aux exportations mondiales (pays à économie planifiée exclus) que les Nations Unies ont établie en subdivisant les exportations en 237 branches, les valeurs de la Suisse sur 1971-1980 sont suffisamment élevées pour figurer dans 70 branches. Or, par rapport à 1973, les parts de 1980 s'inscrivaient à la hausse dans le $52 \%$ des cas et à la baisse dans le $48 \%$ restant. Pour les 35 branches qui en 1976-1977 exportaient en moyenne pour au moins 100.000 dollars de marchandise, ces proportions étaient de $54 \%$ et $46 \%$. Le tableau 7 nous donne la liste des branches en perte de vitesse couvrant ce $48 \%$, à l'exception d'une branche très proche de la stabilité et du secteur de l'armement, dont les fluctuations sont très irrégulières.

II est intéressant de constater que les principaux pays à avoir subtilisé du terrain à l'industrie suisse sont tous des pays développés, à l'exception très importante de l'horlogerie. II est vrai que le tableau 7 n'appréhende environ qu'un tiers des exportations totales, du moins sur la base de 1980. En particulier les critères adoptés ne permettent pas de retenir les branches du secteur textile et de I'habillement, où la pression des NPI est bien connue, comme par ailleurs le tableau 5 nous I'a déjà montré.

\section{CLASSEMENT DES BRANCHES SELON LA CAPACITE CONCURRENTIELLE}

Un classement de ce type nous est livré par Hollenstein $(1979,1980)$, à l'égard de la concurrence des pays à bas salaires. Le classement est fondé sur l'hypothèse que la capacité concurrentielle augmente avec le savoir innovateur, le capital matériel et le capital humain, et qu'elle diminue parallèlement à une augmentation des ressources naturelles. Le secteur industriel est subdivisé en 111 branches qui sont réparties en 5 catégories. Dans la catégorie la plus menacée figurent notamment I'habillement, les chaussures, les articles en cuir et en bois. A l'opposé, dans le groupe qui a le moins à craindre, on trouve en particulier la chimie et la plupart des activités relevant de l'industrie des métaux et des machines. Les montres terminées sont classées dans la catégorie intermédiaire et leurs composantes dans la première. La moitié des personnes occupées en 1977 dans le secteur industriel se trouvaient dans le groupe le moins menacé. De ce classement résulte aussi que le groupe professionnel le plus exposé est celui des travailleurs non qualifiés. $\mathrm{Ce}$ qui confirme les conclusions d'études réalisées pour d'autres pays (5).

Le classement que nous venons d'évoquer peut être comparé à celui, se rapportant toutefois à la capacité concurrentielle vers I'ensemble des autres pays, élaboré par Borner et al. (1978). Dans cette étude, conicernant la période 1968-1977, comme indicateurs de la capacité 
en question on a notamment retenu le taux d'accroissement de la production et de la productivité, le niveau de qualification de la main-d'œuvre employée, l'évolution comparée des prix et des quantités à l'exportation et à l'importation. Sur cette base les 11 branches principales des 13 dans lesquelles est subdivisé habituellement le secteur industriel, sont classées en 4 catégories. Le groupe des branches les plus performantes comprend la chimie et les industries des machines et du caoutchouc. Au deuxième rang figurent la métallurgie, le travail du bois et du verre, l'imprimerie et l'industrie de l'alimentation, boissons et tabac. Au troisième rang il y a l'horlogerie, la production de minerais non ferreux et l'industrie du papier. Enfin, dans la situation la plus critique, on trouve les textiles et I'habillement.

Clairement, les deux études aboutissent à un résultat largement concordant. Pourtant, si on considère l'évolution la plus récente, ces classements doivent être remis en discussion. En effet, comme le fait remarquer le dernier rapport de l'OCDE sur la Suisse (1983), dans la période 1975-1981 la production textile et de l'habilement a progressé de $18 \%$, respectivement de $12 \%$, tandis que le secteur des machines n'a connu qu'un accroissement de $2 \%$ et que la production horlogère a diminué de $28 \%$. En passant, remarquons que l'inversion de tendance des industries du textile et de l'habillement s'est traduite par une amélioration du solde de la balance commerciale de ce secteur, ce qui est unique pour les pays européens à salaires élevés et au regard des années 1973-1979 (6). En tout cas cet exemple, imputable à une politique d'investissements massifs et de spécialisation vers des produits "haut de gamme», témoigne des difficultés de la prévision économique et incite à éviter des scénarios trop rigides.

\section{MARCHE MONDIAL ET POSTES DE TRAVAIL}

Nous avons vu qu'en termes de parts de marché et par rapport aux $\mathrm{NPI}, \mathrm{I}^{\prime}$ industrie suisse a perdu légèrement du terrain. La situation apparaît donc différente de celle qui émerge des seules relations bilatérales (cf. par. 5). Mais cette tendance, en supposant encore qu'elle se confirme, correspond à la logique du développement du Tiers Monde. En effet, elle implique normalement que le taux de croissance du produit national des NPI dépasse celui des pays développés et en particulier de la Suisse: ce qui, au vu des disparités dans le taux de croissance de la population active, est indispensable déjà pour que le revenu par tête progresse au moins au même taux que chez nous. Un tel processus de développement peut augmenter les débouchés des pays développés dans le Tiers Monde et être source de création d'emplois. De toute façon, du changement en question concernant la part des exportations suisses, on ne saurait sans autre déduire, bien qu'on ne puisse pas 
l'exclure, que la concurrence des NPI ait affecté négativement le niveau de l'emploi. Etant donné les chiffres en présence, il convient plutôt d'adhérer à la conclusion de Hollenstein (1980), que globalement les effets sur l'emploi de la concurrence du Tiers Monde ont été négligeables.

Dans l'analyse par branches nous nous bornerons à considérer celles dont l'emploi diminue. Mais d'abord, pour éviter des équivoques, rappelons que le classement des branches d'après la capacité concurrentielle diffère du classement d'après l'indice de l'emploi. En effet un accroissement de la capacité concurrentielle, qui à la longue permet de réaliser un niveau de l'emploi plus élevé qu'autrement, peut très bien dans l'immédiat s'accompagner d'un fléchissement de celui-ci. Ainsi, en particulier, les performances sur le plan de la production de l'industrie textile et de l'habillement se sont traduites sur le plan de l'emploi par une forte réduction.

Si au lieu de la période 1973-1980 nous n'envisageons que les années 1977-1980, de manière à éliminer l'influence de la récession commencée en 1974 et sans que celle de 1982-1983 n'intervienne encore, nous constatons que, $d^{\prime}$ après la subdivision habituelle en treize branches du secteur industriel, les seules branches à avoir enregistré des pertes sensibles d'emplois ont été les textiles, l'habillement et l'horlogerie, c'est-à-dire les branches les plus menacées par le Tiers Monde. La perte d'emploi a été d'environ 13.000 , soit le $8 \%$, tandis qu'en même temps pour l'ensemble de l'industrie I'emploi progressait de $2 \%$. Pour 1973-1980 les mêmes chiffres, cette fois les deux négatifs, sont de 30 et $16 \%$. Sur la même période, si les trois branches n'avaient fléchi que dans la proportion de l'ensemble du secteur, elles auraient conservé environ 30.000 postes supplémentaires. Ce chiffre peut être attribué en grande partie aux pertes de parts de marché ou bien à des mesures visant la hausse de la productivité, toutes deux provoquées par la pression concurrentielle du Tiers Monde.

Pour ce qui concerne en particulier l'industrie horlogère, en revenant au tableau 7 , on remarquera qu'avec la même part de marché qu'en 1973 , en 1980 ses exportations auraient été d'environ $70 \%$ plus élevées. Puisque, au cours de la même période, l'emploi dans I'horlogerie a baissé d'environ $30 \%$ et que les exportations constituent la composante essentielle de ses ventes, cette perte peut être entièrement attribuée à la concurrence étrangère. II y a aussi à signaler que, dans la répartition des points de marché gagnés entre 1973 et 1980, Hongkong, la Corée et Singapour détenaient environ les deux tiers, le tiers restant revenant au Japon (7). L'impact du Tiers Monde sur la suppression des postes de travail dans I'horlogerie peut donc être estimé à tout au plus deux tiers du total, soit environ 17.000 postes de travail. II $s^{\prime}$ agit d'un plafond, car c'est le chiffre valable pour le cas où l'absence de cette concurrence n'aurait pas profité aux autres rivaux, c'est-àdire au Japon. 


\section{RELATIONS AUTRES QUE L'ECHANGE DE MARCHANDISES}

L'impact du Tiers Monde passant par d'autres canaux que l'échange de marchandises a également été évalué par Gerster (1976). Cet auteur prend en compte la balance du tourisme, des activités diplomatiques, des licences et des services restants (banques, assurances, transports, etc.). Pour 1974 et relativement à une définition du Tiers Monde plus large que la nôtre (cf. par. 4), l'effet direct de ces bilans sur l'emploi - qui seraient tous positifs - est estimé à envion 7.000 postes, ce qui correspond au $14 \%$ des postes de travail liés à l'actif de la balance commerciale.

Dans une approche plus complète on devrait aussi envisager l'effet sur l'emploi, lié aux revenus et aux investissements réalisés par la Suisse dans le Tiers Monde. La base statistique est très faible en la matière. Cependant, puisque le flux d'investissements directs et de crédits a presque un caractère unidirectionnel $(8)$, très vraisemblablement le flux des revenus nets est positif. On n'ose pas avoir autant de certitude quant aux autres effets des investissements, du moins dans le cas des investissements directs. Si les investissements ont comme objectif I'accès à un marché fermé par des barrières douanières, I'effet sur l'emploi ne peut être que positif: des nouveaux débouchés s'ouvrent et les investissements entraînent des achats de moyens de production aux pays investisseurs (nous avons déjà tenu compte de cet effet, étant donné qu'il est incorporé dans la balance commerciale); en outre il favorisent la création, dans les maisons mères, de postes de travail relatifs à la gestion des filiales. Si par contre l'objectif réside dans le recours à une main-d'œuvre bon marché, ils peuvent se faire au détriment des investissements, et donc du niveau de l'emploi, domestiques. Les données pour mesurer cet effet de substitution manquent. Contentons-nous de rappeler que des études concernant d'autres pays tendent à montrer que l'effet d'ensemble des investissements directs à l'étranger sur l'emploi domestique est «plutôt positif» (9).

\section{POSTES DE TRAVAIL ET CHOMAGE}

Tout au long de ces notes il a été question de la variation des «postes de travail liés au Tiers Monde». Pour éviter tout malentendu, précisons que cette variation ne correspond pas à une variation du nombre des chômeurs. Le travailleur qui perd sa place dans une branche ne devient pas nécessairement chômeur; il peut aussi quitter la population active (cette situation intéresse en particulier les personnes âgées, les femmes et les travailleurs étrangers rentrant dans leur pays d'origine) ou, 
naturellement, trouver un poste dans une autre branche. Cette dernière possibilité, mais aussi la décision d'arrêter la recherche d'un emploi, seront notamment influencées par la situation conjoncturelle, par les facteurs démographiques qui agissent sur le volume de la population active et en outre par le degré de la mobilité interrégionale et interprofessionnelle. Puisque le premier de ces facteurs est susceptible de varier fortement d'une année à l'autre, il en va de même de la probabilité que la variation des postes de travail liés au Tiers Monde se transforme en chômage.

\section{CONCLUSIONS}

Les études et les statistiques que nous avons prises en compte suggèrent que pour la Suisse, dans la période 1973-1980, le solde du bilan des postes de travail liés au Tiers Monde n'a pas varié sensiblement. Les pertes essuyées par les industries horlogères, de l'habillement et du textile ont été au moins en grande partie compensées par les gains du reste de l'économie. Toutefois si le calcul devait être subdivisé selon les régions et les professions, il laisserait certainement apparaître des déséquilibres, dont ceux qui affectent les régions horlogères sont les plus visibles.

En tout cas, dans l'explication de la réduction nette des postes de travail qui a eu lieu entre 1973 et 1980 , le Tiers Monde ne peut avoir joué qu'un rôle secondaire. Ce rôle devient par contre fondamental, en particulier celui des NPI, si on ne considère que les pertes et pas les gains, et la période intermédiaire entre la fin de la récession de 1974 et le début de celle de 1982-1983.

La variation apparemment peu importante du solde se rapporte par ailleurs à un bilan qui, d'après l'évaluation faite pour 1975, est largement positif. Bilan auquel, $s^{\prime}$ 'il s'agissait de raisonner en termes de bien-être matériel et pas seulement d'emplois, il faudrait ajouter des avantages essentiels provenant de l'extension de la gamme de biens consommés et produits et en outre de la réduction des coûts de production, que permettent les importations depuis le Tiers Monde. Mais sur ce point il est inutile d'insister: si des doutes ont été avancés quant au sens du bilan des relations entre Tiers Monde et Suisse, ils concernent le résultat pour le Tiers Monde, jamais pour la Suisse. 


\section{Notes}

1. Y compris les postes occupés par des personnes travaillant à temps partiel. Source: Annuaire statistique de la Suisse.

2. Cf. Gerster, 1976.

3. Cf. La vie économique, nov. 1980.

4. Cf. par exemple OCDE, 1979.

5. Cf. OCDE, 1979.

6. CF. OCDE, 1983.

7. Cf. Nations Unies, Annuaire statistique du commerce international, 1980, vol. II.

8. Cf. la partie statistique du présent annuaire.

9. Cf. De Laubier, 1982.

\section{Références bibliographiques}

- Borner et al., Structural Analysis of Swiss Industry 1968-1978; Institut für angewandte Wirtschaftsforschung, Universität Basel, 1978 (Discussion Paper no 22).

- De Laubier, D. «Les investissements français vers le Tiers Monde et leur impact sur I'économie française", in Berthelot, Y. et De Bandt, J., "Impact des relations avec le Tiers Monde sur l'économie française", La Documentation française, Paris, 1982.

- Gerster, R. "Schweizerisches Volkseinkommen und Dritte Welt", in Aussenwirtschaft, März 1976.

- Hollenstein, H. Die Auswirkungen der Exportoffensive der Niedriglohnländer auf die Branchenstruktur der schweizerischen Industrie, Diessenhofen, 1979.

- Hollenstein, H. «Die Industrialisierung der EntwicklungsländerRückwirkungen auf die schweizerische Volkswirtschaft", in Revue suisse d'Economie politique de de Statistique, septembre 1980.

- OCDE, L'incidence des nouveaux pays industrialisés, Paris, 1979.

- OCDE, Suisse, mai 1983. 